\title{
Characterization of Primary Direct Acting Antiviral Drugs (DAA) Resistance Mutations in NS5A/NS5B Regions of Hepatitis C Virus with Genotype 1a and $1 \mathrm{~b}$ from Patients with Chronic Hepatitis
}

\author{
Ana Paula de Torres Santos \\ University of Sao Paulo Campus of Sao Paulo: Universidade de Sao Paulo \\ Vanessa Cristina Martins Silva \\ Adolfo Lutz Institute: Instituto Adolfo Lutz \\ Maria Cássia J Mendes-Corrêa \\ Sao Paulo University Faculty of Medicine: Universidade de Sao Paulo Faculdade de Medicina \\ Marcilio Figueiredo Lemos \\ Instituto Adolfo Lutz \\ Fernanda de Mello Malta \\ Albert Einstein Sociedade Beneficente Israelita Brasileira: Hospital Israelita Albert Einstein \\ Rúbia Anita Ferraz Santana \\ Albert Einstein Israelite Hospital: Hospital Israelita Albert Einstein \\ Gregório Tadeu Fernando Dastoli \\ Albert Einstein Sociedade Beneficente Israelita Brasileira: Hospital Israelita Albert Einstein \\ Vanessa Fusco Duarte de Castro \\ Albert Einstein Sociedade Beneficente Israelita Brasileira: Hospital Israelita Albert Einstein \\ João Renato Rebello Pinho \\ Albert Einstein Sociedade Beneficente Israelita Brasileira: Hospital Israelita Albert Einstein and University of Sao Paulo \\ Regina Célia Moreira ( $\nabla$ regina.moreira7@gmail.com) \\ Instituto Adolfo Lutz https://orcid.org/0000-0002-8208-8776
}

\section{Research Article}

Keywords: DAA, Hepatitis C virus, Genotype 1a and 1b, RAS, NS5B, NS5A

Posted Date: June 21st, 2021

DOI: https://doi.org/10.21203/rs.3.rs-529088/v1

License: (c) (1) This work is licensed under a Creative Commons Attribution 4.0 International License. Read Full License 


\section{Abstract}

Hepatitis $\mathrm{C}$ virus ( $\mathrm{HCV}$ ) infection is a public health problem with an estimated 71 million infected people worldwide. The high level of HCV replication and its lack of post - transcriptional correction mechanisms result in the rapid emergence of viral variants, difficulty in determining polymorphisms, and variants that contain substitutions associated with resistance and/or reduction of susceptibility towards new antivirals. The aim of this study was to map the polymorphisms in NS5A and NS5B and resistance mutations to new antiviral drugs in HCV strains with genotype 1a and 1b derived from patients with chronic hepatitis $\mathrm{C}$ infection. Serum samples from patients who underwent routine viral load tests and monitoring at the laboratory of viral hepatitis at the Adolfo Lutz Institute, São Paulo, Brazil were collected. A total of 698 and 853 samples were used for the characterization of NS5A and NS5B regions respectively; comprising HCV genotypes $1 \mathrm{a}$ and $1 \mathrm{~b}$. The prevalence of resistance mutations in the NS5A region was found to be $6.4 \%$, with $\mathrm{Y} 93 \mathrm{H}, \mathrm{L} 31 \mathrm{M}, \mathrm{Q} 30 \mathrm{R}$, and $\mathrm{Y} 93 \mathrm{~N}$ as the main resistance-associated substitutions (RAS). No NS5B- associated RAS was observed for any of the drugs analyzed. This study reveals the presence of significant RAS in the HCV serum samples. These findings support the RAS test should be offered to individuals with poor response to double combination regimens prior to treatment initiation, thereby assisting strain vigilance and selection of effective treatment or retreatment options using DAA regimens.

\section{Introduction}

Hepatitis $\mathrm{C}$ virus (HCV) is a serious public health problem, affecting approximately 71 million people worldwide [1]. HCV is classified into eight genotypes (GT) with approximately $30 \%$ variability, and 86 subtypes that have been described with $20 \%$ variability [2]. In Brazil, HCV genotypes $1 \mathrm{a}$ and $1 \mathrm{~b}$ are the most common, prevalent in approximately $64.9 \%$ of all the patients infected with HCV, followed by GT $3(30.2 \%)$. On the other hand; the GT 2,4 , and 5 are found less frequently in Brazil with $4.6 \%, 0.2 \%$, and $0.1 \%$ prevalence, respectively [3].

Fortunately, the development of direct-acting antivirals (DAAs) has reached a sustained virological response (SVR) with successful treatment in nearly $95 \%$ of patients with HCV and with few adverse events [4]. The high level of HCV replication and its lack of post-transcriptional correction mechanisms result in the rapid emergence of viral variants in quasispecies. These factors are responsible for determining not only the viral polymorphisms, but also the variants that contain substitutions associated with resistance and/or reduction of susceptibility to DAAs $[5,6]$.

The current study highlights NS5B polymerase and NS5A inhibitors, as classes of drugs used in treatment, which are available at Sistema Único de Saúde SUS in Brazil (Unified National Health System) [7].

Despite the overall success, antiviral treatment for certain groups of patients remains a challenge. For some patients, Ribavirin is still recommended; as it improves the rate of SVR, especially in patients with advanced liver diseases [8].

NS5A inhibitors quickly select resistant variants when administered alone, as they have a low genetic barrier to resistance. Among the main mutations in the NS5A region that impart high levels of resistance to NS5A inhibitors, the most common are Q30E/H/K, Y93N/C, L31M, and Y93H [9]. In contrast, despite having a high genetic barrier; some substitutions in NS5B have been associated with resistance to Sofosbuvir [10]. As described by Jiménez-Pérez et al., some compensatory mutations that can recover the capacity for viral replication in presence of certain mutations have also been described [11].

The real impact of resistance mutations that are relevant in the treatment-naïve patients on the effectiveness of treatment, are issues that still need to be clarified. This study aimed to characterize primary resistance mutations and map the RAS in NS5A and NS5B regions in the HCV-1a and - $1 \mathrm{~b}$ genotypes from naïve patients with chronic hepatitis C DAA in the Brazilian state of São Paulo.

\section{Materials And Methods}

\section{Sampling analyzed}

Samples from patients belonging to different regions of São Paulo State, including Vale do Paraíba, Vale do Ribeira, and São Paulo Metropolitan area, which included São Paulo and ABCD regions; were included in this study. These serum samples were from DAA-naïve patients chronically infected with HCV GT-1a and GT-1b, and were sent to Adolfo Lutz Institute (IAL), a public health laboratory in São Paulo state of Brazil, from 2012 to 2014 . Patient samples were used for HCV diagnosis and stored at $-20^{\circ} \mathrm{C}$.

\section{Amplification and sequencing of NS5A and NS5B regions}

Nucleic acid extraction was performed using the NucliSENS easyMAG ${ }^{\mathrm{TM}}$ kit (BioMérieux, Marcy l'Etoile, France), as per the manufacturer's instructions.

The amplification of HCV NS5A region was performed in a single step using the SuperScript ${ }^{T M}$ III One-Step RT-PCR System with Platinum ${ }^{\text {TM }}$ Taq DNA Polymerase (Invitrogen ${ }^{\circledR}$, Thermo Fisher, Carlsbad, USA), and a set of primers described in Table 1 [12]. PCR products were obtained using the following thermal cycling conditions: $50^{\circ} \mathrm{C}$ for $30 \mathrm{~min}$ and $94^{\circ} \mathrm{C}$ for $3 \mathrm{~min}$, followed by 45 cycles of $94{ }^{\circ} \mathrm{C}$ for $15 \mathrm{~s}, 48^{\circ} \mathrm{C}+0.3^{\circ} \mathrm{C} / \mathrm{cycle}$ for $30 \mathrm{~s}, 68^{\circ} \mathrm{C}$ for $1 \mathrm{~min}$ and 68 ${ }^{\circ} \mathrm{C}$ for 5 min.

The amplification of HCV NS5B region was performed in two steps using the protocol described by Santos et al [13], and a set of primers described in Table 1 [14]. For both regions, amplified fragments were visualized by electrophoresis, using $2 \%$ agarose gel and SYBR Safe DNA gel stain (Invitrogen ${ }^{\mathrm{TM}}$, Carlsbad, CA, USA).

Samples were sequenced by Sanger method [15], using commercial Kits, according to the protocol described by Santos et al. [13]. To characterize genotypes and identify polymorphisms, the sequences were submitted to Geno2Pheno site (https://hcv.geno2pheno.org). 


\section{Results}

\section{Study population}

For the characterization of NS5A region, a total of 698 samples were analyzed; of which 456 patients (65.3\%) were from the São Paulo metropolitan area and 242 (34.7\%) from Vale do Paraiba or Vale do Ribeira, Brazil. For NS5B characterization, a total of 853 samples were analyzed; of which 541 patients (63.4\%) were from the São Paulo metropolitan area and 312 (36.6\%) from Vale do Paraiba or Vale do Ribeira.

Of the 698 samples used for NS5A region characterization, 305 (43.7\%) belonged to GT-1a and 393 (56.3\%) to GT-1b. Of the total, 371 (53.2\%) and 327 (46.8\%) were men and women respectively. Of the 853 samples used for NS5B region characterization, 456 ( $53.5 \%$ ) belonged to GT-1a, and 397 ( $46.5 \%$ ) belonged to GT-1b. Of the total, 458 (53.7\%) and 395 (46.3\%) were men and women respectively.

\section{Characterization of polymorphisms for NS5A and NS5B regions}

A total of 698 samples of HCV GT-1a and GT-1b were used for characterizing the NS5A region. The results revealed absence of polymorphism in 531 (76.1\%) samples, natural polymorphism in $116(16.6 \%)$ samples, 6 (0.9\%) samples with reduced susceptibility, and 45 (6.4\%) samples exhibiting RAS at baseline. The most frequent age group that showed RAS was in the range of 40-59 years (Table 2).

Some relevant RAS were observed in 45 (6.4\%) out of the 698 samples analyzed for NS5A region characterization. The most prevalent polymorphisms observed were $\mathrm{Y} 93 \mathrm{H}, \mathrm{L} 31 \mathrm{M}, \mathrm{Q} 30 \mathrm{R}$, and $\mathrm{Y} 93 \mathrm{~N}$; in isolation or association with other polymorphisms. Of the 45 samples demonstrating the presence of RAS, 13 and 32 belonged to GT-1a and GT-1b respectively. The most frequent RAS for GT-1a was L31M, followed by Q30R and Y93N. For GT-1b, the most frequent RAS was $\mathrm{Y} 93 \mathrm{H}$, followed by L31M.

Among the mutations in the NS5A region detected for Daclatasvir resistance, the most frequent RAS was Y93H (62.5\%); followed by L31M (12.5\%) and Q30R (9.4\%). For Elbasvir resistance, Y93H was also the most frequent RAS (52.4\%), followed by L31M (28.6\%). Ledipasvir resistance presented RAS Y93H in 43.9\% and L31M in $26.9 \%$ of patients. For Velpatasvir resistance, RAS Y $93 \mathrm{H}$ was observed as an isolated polymorphism in $88 \%$ of the samples and as an associated polymorphism with $\mathrm{Q} 30 \mathrm{H}$ in $4.0 \%$. Y93N polymorphism was detected at a concentration of $8.0 \%$. For Pibrentasvir resistance, only two samples showed RAS with the Y93N polymorphism. Ombitasvir resistance demonstrated $\mathrm{Y} 93 \mathrm{H}$ in $70 \%$ of the samples and Q30R in $10.0 \%$. However, other polymorphisms were observed at a lower rate. These data are presented in Table 3. Table 4 shows the frequency of RAS for each DAA in the NS5A region.

In this study, none of the 853 samples analyzed for polymorphisms in NS5B region of HCV GT-1a and GT-1b presented RAS that confer resistance to Sofosbuvir.

\section{Discussion}

Since 2014, DAAs have been used for the treatment of chronic Hepatitis C in Brazil. They are dispensable freely for all patients but drugs are controlled by Ministry of Health (Brazil). To ensure that the patients have reached the SVR, the absence of HCV-RNA must be observed six months after the treatment has already finished [7].

Adolfo Lutz Institute (IAL) in São Paulo City, Brazil has played an important role in diagnosing viral load and monitoring patients from public health services, starting from diagnosis to analysis of virological sustained response. In addition, IAL monitors the appearance of resistance mutations in these patients.

There is no gold standard available to detect HCV drug resistance so far. In this study, the Sanger sequencing method was employed for the detection of mutations associated with antiviral drug resistance associated with HCV [15], as a tool with great potential for application in a public health diagnostic laboratory. Many studies on HCV RAS have used this methodology to detect important polymorphisms, as it has a low cost and is easier to perform [13,16,17].

All the patients enrolled in this study were treatment-naïve until the samples were collected to process for the molecular tests. The population examined represents the patients with most chronic HCV in Brazil who were awaiting treatment. The population was heterogeneous, composed mainly of male individuals who had a high viral load, were approximately 50 years of age and from different regions of the state of São Paulo, Brazil [13,18,19].

The distribution of HCV GTs varies according to geographic location, and monitoring this distribution is important to define epidemiological trends of infection regarding the introduction of new GTs and determining transmission routes [20, 21]. In this study, samples belonging to GT-1 were evaluated to determine the frequency of DAA-resistant variants. The available drugs for the treatment of HCV GT-1 include Daclatasvir, Elbasvir, Ledipasvir, Ombitasvir, Pibrentasvir, Velpatasvir, Sofosbuvir, and Dasabuvir. For the GT-1 NS5A region, our study revealed the presence of RAS in $6.4 \%$ out of a total of 698 samples analyzed. Previous studies have indicated the frequency of RAS in the NS5A region of HCV, determined by conventional and next- generation sequencing assays, to be between $6.0 \%$ and $16.0 \%[22,23]$. The prevalence of NS5A RAS is 18.0 to $21.0 \%$ in Asian countries, $15.0 \%$ in North America, and $19.0 \%$ in Europe. The prevalence of NS5B RASs is $1.0 \%$ to $5.0 \%, 4.0 \%$ and $20.0 \%$ in Asian countries, North America, and Europe respectively [24].

This study shows that patients with HCV GT-1 already have some RAS at baseline. The most prevalent polymorphisms observed in GT-1 are Y93H, L31M, Q30R, and Y93N, existing in isolation or in association with another polymorphism. These polymorphisms were also reported by Rao et al. in China [24]. In samples that presented RAS, a significant association was observed with GT-1b (71.1\%), as opposed to GT-1a (28.9\%). Previous studies suggest that GT-1b may accumulate more resistance mutations than the GT-1a strain to overcome the action of DAAs [25]. The most frequent RAS found for GT-1a was L31M, followed by Q30R and Y93N whereas for GT-1b; it was Y93H followed by L31M. The single mutation of Y93H or Q30R confers high levels of Ledipasvir resistance to both HCV strains with GT-1a and GT1b. For GT-1b, this resistance was observed after a short period of treatment; whereas for GT-1a, it was only observed in patients who received high doses [26]. 
According to Cuypers et al., the most important RAS that interfere with the effectiveness of NS5A inhibitors are M/L28T/V, Q/L30E/H/R/S, L31M/V, H58D, and $\mathrm{Y} 93 \mathrm{C} / \mathrm{H} / \mathrm{N}$, which reduce susceptibility to NS5A inhibitors and induce conformational changes [27]. In addition, Gottwein et al. have demonstrated that the $\mathrm{Y} 93 \mathrm{H}$ polymorphism shows clinical results on the effectiveness of Pibrentasvir [28]. As a result, the mutation $\mathrm{Y} 93 \mathrm{H}$ may well be responsible for selection of resistant strains in the NS5A region, which may interfere with treatment based on DAAs [16, 28].

The most frequent mutations in GT-1 were Y93H and L31M. Of all the samples analyzed, the resistance to Daclatasvir and Ledipasvir with L31M was observed in 4 and 11 samples respectively. The $\mathrm{Y} 93 \mathrm{H}$ mutation was observed in 20 and 18 samples that presented resistance to Daclatasvir and Ledipasvir respectively. A study carried out in vitro by Wyles and Luetkemeyer, demonstrated that the substitution of the amino acid L31M is more likely confers a clinical impact due to the high fold change observed in vitro towards Daclatasvir and Ledipasvir sensitivity [29].

However, an in vivo study by Costa et al. has indicated that the basal presence of RAS L31M and Y93H does not influence the treatment results, since all patients with this mutation have already reached SVR [30].

In our study, two samples that showed the polymorphism Y93N also presented resistance to Pibrentasvir. However, a study performed by Wyles \& Luetkemeyer has shown that this mutation does not seem to have a significant clinical impact with respect to this drug, due to the low fold-change (<10X for GT-1a), but, due to the high fold-change, this polymorphism is more likely to confer a clinical impact for other drugs like Elbasvir, Velpatasvir (>1000X for GT-1a), Daclatasvir, Ledipasvir and Ombitasvir (>10,000X for GT-1a) [29]. The absence of RAS in the NS5B region was observed in all the analyzed sequences. Polymerase inhibitors have a high genetic barrier; therefore, only a few patients experience therapeutic failure with the Sofosbuvir regimen [31]. The persistence of NS5A RAS has been well described and can be selected by long-term immune pressure or other factors that are still undetermined [32, 33].

Unfortunately, the samples used for this study were not sent with clinical information. Hence, it was not possible to carry out a comparative study between RAS found in this study and those that caused effective post-treatment resistance. In addition, due to a lack of complete data and clinical follow-up of patients, it was not possible to evaluate whether these patients had SVR. A study with the clinical follow-up of patients would be important to understand whether the mutations found in the laboratory tests cause effective resistance to drugs, as an inappropriate choice of drugs together with a short duration or poor adherence to treatment are important factors that can influence treatment failure in real life. A study performed by Chen et al. has identified the lack of treatment adherence as the main failure factor in SVR. The negative impact of this factor can be underestimated due to a lack of pre-treatment counseling that reinforces the importance of adherence for successful treatment in practice [34].

The prevalence of a baseline RAS determined in this study aligns with that reported in other countries [22-24]. The NS5A region, as expected, is more susceptible to RAS than the NS5B region, corroborating the conclusions of other studies highlighting that the NS5B region of the HCV genome represents a high barrier to mutations. However, these findings should be supported by further studies including the clinical follow-up of patients and the comparison between the mutations found in laboratory tests and those that cause effective drug resistance. Such additional information is essential to allow the choice of drug alternatives for the treatment or retreatment of patients with chronic hepatitis GT-1a and GT- $1 \mathrm{~b}$.

Overall, here, we reveal that the prevalence of RAS in the NS5A region of HCV is $6.4 \%$, in the context of chronic Hepatitis C patients from important regions of the São Paulo State. Importantly, this prevalence may be behind the failure to achieve virological sustained response in some cases. Although pangenotypic drugs are highly efficacious options for the retreatment of chronic HCV patients, our findings support the notion that the RAS test should be offered to individuals with poor response to double combination regimens prior to treatment initiation.

\section{Declarations}

Conflict of interest. All authors declare that they have no conflicts of interest or disclosures to the manuscript.

Ethical standards: All the proceedings of this study were only initiated post obtaining ethical approvals from relevant ethics committees of the participating institutions. This study was approved by the Ethical Committee in Research at the Adolfo Lutz Institute, Brazil (CEPIAL) \# 1.040.338.

Data availability statement: The data that support the findings of this study are available from the corresponding author upon reasonable request, but clinical data or other information from patients enrolled in this study, are not available.

Funding: This study was supported by the Fundação de Amparo à Pesquisa do Estado de São Paulo (FAPESP: \# 2017/01809-9 and \#2020/12529-0).

Acknowledgments: We are grateful to Fundação de Amparo à Pesquisa do Estado de São Paulo for funding this research.

\section{References}

1. World Health Organization (WHO) (2017) Global hepatitis report 2017. Geneva. Available from: https://apps.who.int/iris/bitstream/handle/10665/255016/9789241565455-eng.pdf. Accessed 15 march 2021.

2. Borgia SM, Hedskog C, Parhy B, et al. (2018) Identification of a novel hepatitis C virus genotype from Punjab, India: expanding classification of hepatitis C virus into 8 genotypes. J Infect Dis 218(11):1722-1729. doi: 10.1093/infdis/jiy401.

3. Campiotto S, Pinho JRR, Carrilho FJ, et al. (2005) Geographic distribution of hepatitis C virus genotypes in Brazil. Braz J Med Biol Res 38(1):41-49. doi: https://dx.doi.org/10.1590/S0100-879X2005000100007. 
4. Liu Z, Mao X, Yu K, et al. (2020) Prevalence of HCV resistance-associated substitutions among treatment-failure patients receiving direct-acting antiviral agents. J Viral Hepat. 27(6):585-592. doi: 10.1111/jvh.13270.

5. Kim CW, Chang KM (2013) Hepatitis C virus: virology and life cycle. Clin Mol Hepatol 19(1):17-25. doi: 10.3350/cmh.2013.19.1.17.

6. Scheel TKD, Rice CM (2013) Understanding the hepatitis C virus life cycle paves the way for highly effective therapies. Nat Med 19(7):837-849. doi: $10.1038 / \mathrm{nm} .3248$.

7. Ministry of Health (MS). (2019) Clinical Protocol and Therapeutic Guidelines for Hepatitis C and Co-infections. Brasília-DF, 72p. Available from: http://www.aids.gov.br/pt-br/pub/2017/protocolo-clinico-e-diretrizes-terapeuticas-para-hepatite-c-e-coinfeccoes. Accessed 21 march 2021.

8. Foster GR, Irving WL, Cheung MC, et al. (2016) Impact of direct acting antiviral therapy in patients with chronic hepatitis C and decompensated cirrhosis. J Hepatol 64(6):1224-1231. doi: 10.1016/j.jhep.2016.01.029.

9. Paolucci S, Fiorina L, Mariani B, Gulminetti R, Novati S, Barbarini G et al. (2012) Naturally occurring mutations to HCV protease inhibitors in treatment-naïve patients. Virol j (9):245. doi: https://doi.org/10.1186/1743-422X-9-245.

10. Hedskog C, Dvory-Sobol H, Gontcharova V, Martin R, Ouyang W, Han B et al. (2015) Evolution of the HCV viral population from a patient with S282T detected at relapse after sofosbuvir monotherapy. J Viral Hepat 22:871-881. doi: 10.1111/jvh.12405.

11. Jiménez-Pérez M, González-Grande R, Contreras PE, Martínez IP, Lombardo JC, Martín RO (2016) Treatment of chronic hepatitis C with direct-acting antivirals: The role of resistance. World J Gastroenterol 22(29):6573-6581. doi: 10.3748/wjg.v22.i29.6573.

12. Andre-Garnier E, Besse B, Rodallec A, et al. (2017) An NS5A single optimized method to determine genotype, subtype and resistance profiles of Hepatitis $C$ strains. PLOS ONE 12(7): e0179562. doi: https://doi.org/10.1371/journal.pone.0179562.

13. Santos APT, Silva VCM, Mendes-Corrêa MCJ, et al. (2021) Prevalence and Pattern of Resistance in NS5A/NS5B in Hepatitis C Chronic Patients Genotype 3 Examined at a Public Health Laboratory in the State of São Paulo, Brazil. Infect Drug Resist 14:723-730. doi:10.2147/IDR.S247071.

14. Sandres-Sauné K, Deny P, Pasquier C, Thibaut V, Duverlie G, Izopet J (2003) Determining hepatitis C genotype by analyzing the sequence of the NS5b region. J Virol Methods 109(2):187-193. doi:10.1016/s0166-0934(03)00070-3.

15. Sanger F, Nicklen S, Coulson AR (1977) DNA sequencing with chain-terminating inhibitors. Proc Natl Acad Sci USA 74(12):5463-5467.

16. Sharafi H, Alavian SM (2018) Hepatitis C resistance to NS5A inhibitors: Is it going to be a problem? World J Hepatol 10(9):543-548. doi: 10.4254/wjh.v10.i9.543.

17. Sarrazin C (2016) The importance of resistance to direct antiviral drugs in HCV infection in clinical practice. J Hepatol 64(2):486-504. doi.10.1016/j.jhep.2015.09.011.

18. Gower E, Estes C, Blach S, Razavi-Shearer K, Razavi H (2014) Global epidemiology and genotype distribution of the hepatitis C virus infection. J Hepatol 61(1suppl):S45-S57. doi:10.1016/j. jhep.2014.07.027.

19. Sorbo MC, Cento V, Di Maio VC, et al. (2018) Hepatitis C virus drug resistance associated substitutions and their clinical relevance. Drug Resist Updat 37:17-39. doi:10.1016/j.drup.2018.01.004.

20. Simmonds P, Bukh J, Combet C, et al. (2005) Consensus proposals for a unified system of nomenclature of hepatitis C virus genotypes. Hepatology 42:962-973. doi: 10.1002/hep.20819.

21. Lavanchy D (2011) Evolving epidemiology of hepatitis C virus. Clin Microbiol Infect 17(2):107-115. doi: 10.1111/j.1469-0691.2010.03432.x.

22. Suzuki F, Sezaki H, Akuta N, et al. (2012) Prevalence of hepatitis C virus variants resistant to NS3 protease inhibitors or the NS5A inhibitor (BMS-790052) in hepatitis patients with genotype 1b. J Clin Virol 54(4):352-354. doi: 10.1016/j.jcv.2012.04.024.

23. Sarrazin C, Berg T, Buggisch P, et al. (2014) Current recommendations for the treatment of chronic hepatitis C. Z Gastroenterol 52(10):1185-1197.

24. Rao H, Yang X, Tan Y, et al. (2020) Efficacy and Safety of All-oral Emitasvir and Sofosbuvir in Patients with Genotype 1b HCV Infections without Cirrhosis. J Clin Transl Hepatol 8(3):255-261. doi:10.14218/JCTH.2020.00031

25. Sulkowski MS, Gardiner DF, Rodriguez-Torres M, et al. (2014) Daclatasvir plus sofosbuvir for previously treated or untreated chronic HCV infection. N Engl J Med 370(3):211-221. doi: 10.1056/nejmoa1306218.

26. Cheng G, Tian Y, Doehle B, et al. (2016) In vitro antiviral activity and resistance profile characterization of the hepatitis C virus NS5A inhibitor ledipasvir. Antimicrob Agents Chemother 60:1847-1853. doi: 10.1128/AAC.02524-15.

27. Cuypers L, Ceccherini-Silberstein F, Van Laethem K, Li G, Vandamme AM, Rockstroh JK (2016) Impact of HCV genotype on treatment regimens and drug resistance: a snapshot in time. Rev Med Virol 26(6):408-434. doi: 10.1002/rmv.1895. 
28. Gottwein JM, Pham LV, Mikkelsen LS, et al. (2018) Efficacy of NS5A Inhibitors Against Hepatitis C Virus Genotypes 1-7 and Escape Variants. Gastroenterology 154(5):1435-1448. doi: 10.1053/j.gastro.2017.12.015.

29. Wyles DL, Luetkemeyer AF (2017) Understanding Hepatitis C Virus Drug Resistance: Clinical Implications for Current and Future Regimens. Top antivi med 25(3):103-109.

30. Costa VD, Brandão-Mello CE, Nunes EP, et al. (2019) Treatment of chronic HCV infection with DAAs in Rio de Janeiro/Brazil: SVR rates and baseline resistance analyses in NS5A and NS5B genes. PLoS One 14(5):e0216327. doi: https://doi.org/10.1371/journal.pone.0216327.

31. Sagnelli E, Starace M, Minichini C, et al. (2018) Resistance detection and re-treatment options in hepatitis C virus-related chronic liver diseases after DAAtreatment failure. Infection 46(6):761-783. doi: 10.1007/s15010-018-1188-3.

32. Eltahla AA, Leung P, Pirozyan MR, et al. (2017) Dynamic evolution of hepatitis $C$ virus resistance-associated substitutions in the absence of antiviral treatment. Sci Rep 7:41719. doi: https://doi.org/10.1038/srep41719.

33. Yoshimi S, Imamura M, Murakami E, et al. (2015) Long term persistence of NS5A inhibitor-resistant hepatitis C virus in patients who failed daclatasvir and asunaprevir therapy. J Med Virol 87:1913e20. doi: 10.1002/jmv.24255.

34. Chen CY, Huang CF, Cheng PN, et al. (2021) Factors associated with treatment failure of direct-acting antivirals for chronic hepatitis C: A real-world nationwide hepatitis $C$ virus registry programme in Taiwan. Liver Int Online ahead of print. epub.PMID: 33655714. doi: 10.1111/liv.14849.

\section{Tables}

Table 1: Sequence of primers used for GT1a and 1b for regions NS5A and NS5B. Adapted from Santos et al. [13].

\begin{tabular}{|lllll|}
\hline Region & Direction & Sequence $\left(\mathbf{5}^{\prime} \mathbf{-} \mathbf{3}^{\prime}\right)$ & Position & Reference \\
\hline NS5A: & & & & \\
\hline 2F & Sense & 5'ACTGTAAAACGACGGCCAGTGGIGARGGIGCIGTICARTGGATGAA3' & $6066-6091$ & {$[12]$} \\
\hline R & Antisense & 5'ACCAGGAAACAGCTATGACCTRTGRGAIGGRTCIGTIARCATIGA3' & $6882-6858$ \\
\hline 3R & Antisense & 5'ACCAGGAAACAGCTATGACCTRTGRGAIGGRTCICTIARCATIGA3' & $6882-6858$ \\
\hline NS5B: & & & & \\
\hline PR1 & Sense & 5'TGGGGATCCCGTATGATACCCGCTGCTTTGA3' & $8245-8275$ & {$[14]$} \\
\hline PR2 & Antisense & 5'GGCGGAATTCCTGGTCATAGCCTCCGTGAA3' & $8616-8645$ \\
\hline PR3 & Sense & 5'TATGAYACCCCCTGYTTTGACTC3' & $8256-8278$ \\
\hline PR5 & Antisense & 5'GCTAGTCATAGCCTCCGT 3' & $8619-8636$ \\
\hline
\end{tabular}

Table 2: Characteristics of the patients who participated in this study according to the samples sequence results for NS5A region ( $n=698$ ). Adapted from Santos et al. [13]. 


\begin{tabular}{|c|c|c|c|c|c|}
\hline & \multicolumn{5}{|c|}{ Samples sequence for NS5A region } \\
\hline & $\begin{array}{l}\text { Samples without } \\
\text { RAS* }\end{array}$ & $\begin{array}{l}\text { Samples with } \\
\text { Polymorphisms }\end{array}$ & $\begin{array}{l}\text { Samples with reduced } \\
\text { susceptibility }\end{array}$ & $\begin{array}{l}\text { Samples with } \\
\text { RAS }\end{array}$ & Total \\
\hline \multicolumn{6}{|l|}{ Gender } \\
\hline Female & 253 & 53 & 3 & 18 & 327 \\
\hline Male & 278 & 63 & 3 & 27 & 371 \\
\hline \multicolumn{6}{|l|}{ Age (years) } \\
\hline $1-09$ & 2 & 0 & 0 & 0 & 2 \\
\hline $10-19$ & 3 & 1 & 0 & 0 & 4 \\
\hline $20-29$ & 26 & 4 & 0 & 0 & 30 \\
\hline $30-39$ & 97 & 23 & 0 & 11 & 131 \\
\hline $40-49$ & 160 & 41 & 1 & 16 & 218 \\
\hline $50-59$ & 125 & 26 & 3 & 9 & 163 \\
\hline $60-69$ & 94 & 13 & 2 & 8 & 117 \\
\hline $70-79$ & 21 & 8 & 0 & 1 & 30 \\
\hline$>80$ & 3 & 0 & 0 & 0 & 3 \\
\hline \multicolumn{6}{|c|}{ Regions of São Paulo State } \\
\hline $\begin{array}{l}\text { São Paulo Metropolitan } \\
\text { area }\end{array}$ & 343 & 80 & 4 & 29 & 456 \\
\hline Vale Paraíba/Ribeira & 188 & 36 & 2 & 16 & 242 \\
\hline \multicolumn{6}{|l|}{ Genotype } \\
\hline $1 \mathrm{a}$ & 224 & 64 & 4 & 13 & 305 \\
\hline $1 b$ & 307 & 52 & 2 & 32 & 393 \\
\hline
\end{tabular}

* Resistance associated substitutions

Table 3: Distribution of polymorphisms found for the HCV NS5A region, according to the DAA $(n=45)$. Adapted from Santos et al. [13]. 


\begin{tabular}{|c|c|c|c|c|c|c|c|c|c|c|}
\hline \multicolumn{11}{|l|}{ Drugs } \\
\hline Sample & Genotype & Polymorphism & Daclatasvir & Polymorphism & Elbasvir & Polymorphism & Ledipasvir & Polymorphism & Ombitasvir & Polyn \\
\hline 73 & $1 \mathrm{a}$ & L31M & $\mathrm{R}$ & L31M & $\mathrm{R}$ & L31M & $\mathrm{R}$ & L31M & SB & L31M \\
\hline 95 & $1 b$ & $\mathrm{Y93H}$ & $\mathrm{R}$ & $\mathrm{Y93H}$ & $\mathrm{R}$ & $\mathrm{Y93H}$ & $\mathrm{R}$ & Y93H & $\mathrm{R}$ & / \\
\hline 108 & $1 b$ & Y93H & $\mathrm{R}$ & Y93H & $\mathrm{R}$ & Y93H & $\mathrm{R}$ & Y93H & $\mathrm{R}$ & I \\
\hline 142 & $1 b$ & Y93H & $\mathrm{R}$ & Y93H & $\mathrm{R}$ & Y93H & $\mathrm{R}$ & Y93H & $\mathrm{R}$ & / \\
\hline 160 & $1 \mathrm{a}$ & Y93N & $\mathrm{R}$ & Y93N & $\mathrm{R}$ & Y93N & $\mathrm{R}$ & Y93N & $\mathrm{R}$ & Y93N \\
\hline 195 & $1 b$ & $\mathrm{Y93H}$ & $\mathrm{R}$ & Y93H & $\mathrm{R}$ & Y93H & $\mathrm{R}$ & Y93H & $\mathrm{R}$ & / \\
\hline 254 & $1 a$ & Q30R & $\mathrm{R}$ & Q30R & $\mathrm{R}$ & Q30R & $\mathrm{R}$ & Q30R & $\mathrm{R}$ & Q30R \\
\hline 255 & $1 b$ & Y93H & $\mathrm{R}$ & Y93H & $\mathrm{R}$ & Y93H & $\mathrm{R}$ & Y93H & $\mathrm{R}$ & I \\
\hline 279 & $1 b$ & L31M & RS & L31M & $\mathrm{R}$ & L31M & $\mathrm{R}$ & L31M & SB & / \\
\hline 285 & $1 \mathrm{a}$ & L31M & $\mathrm{R}$ & L31M & $\mathrm{R}$ & L31M & $\mathrm{R}$ & L31M & SB & L31M \\
\hline 289 & $1 b$ & А92T,Y93H & $\mathrm{R}$ & $\mathrm{Y93H}$ & $\mathrm{R}$ & A92T,Y93H & $\mathrm{R}$ & Y93H & $\mathrm{R}$ & / \\
\hline 302 & $1 b$ & А92T,Y93H & $\mathrm{R}$ & Y93H & $\mathrm{R}$ & A92T,Y93H & $\mathrm{R}$ & Y93H & $\mathrm{R}$ & / \\
\hline 308 & $1 b$ & Y93H & $\mathrm{R}$ & Y93H & $\mathrm{R}$ & Y93H & $\mathrm{R}$ & Y93H & $\mathrm{R}$ & l \\
\hline 355 & $1 b$ & L31M & RS & L31M & $\mathrm{R}$ & L31M & $\mathrm{R}$ & L31M & SB & I \\
\hline 410 & $1 b$ & $\mathrm{Y93H}$ & $\mathrm{R}$ & $\mathrm{Y93H}$ & $\mathrm{R}$ & P58?,Y93H & $\mathrm{R}$ & Y93H & $\mathrm{R}$ & / \\
\hline 455 & $1 b$ & L31M & RS & L31M & $\mathrm{R}$ & L31M & $\mathrm{R}$ & L31M & SB & / \\
\hline 458 & $1 b$ & Y93H & $\mathrm{R}$ & Y93H & $\mathrm{R}$ & Y93H & $\mathrm{R}$ & Y93H & $\mathrm{R}$ & / \\
\hline 462 & $1 b$ & L31M & RS & L31M & $\mathrm{R}$ & L31M,P58S & $\mathrm{R}$ & L31M & SB & / \\
\hline 491 & $1 \mathrm{a}$ & Y93S & SB & Y93S & SB & Y93S & SB & Y93S & $\mathrm{R}$ & Y93S \\
\hline 497 & $1 b$ & Y93H & $\mathrm{R}$ & Y93H & $\mathrm{R}$ & Y93H & $\mathrm{R}$ & Y93H & $\mathrm{R}$ & I \\
\hline 577 & $1 \mathrm{a}$ & Y93N & $\mathrm{R}$ & Y93N & $\mathrm{R}$ & Y93N & $\mathrm{R}$ & Y93N & $\mathrm{R}$ & Y93N \\
\hline 602 & $1 b$ & Y93N & $\mathrm{R}$ & Y93N & $\mathrm{R}$ & Y93N & $\mathrm{R}$ & Y93N & $\mathrm{R}$ & / \\
\hline 611 & $1 b$ & L31F & SB & L31F & $\mathrm{R}$ & L31F & SB & L31F & RS & I \\
\hline 641 & $1 b$ & L31M & RS & L31M & $\mathrm{R}$ & L31M & $\mathrm{R}$ & L31M & SB & / \\
\hline 645 & $1 b$ & Y93H & $\mathrm{R}$ & Y93H & $\mathrm{R}$ & Y93H & $\mathrm{R}$ & Y93H & $\mathrm{R}$ & I \\
\hline 652 & $1 b$ & Y93H & $\mathrm{R}$ & Y93H & $\mathrm{R}$ & Y93H & $\mathrm{R}$ & Y93H & $\mathrm{R}$ & / \\
\hline 660 & $1 b$ & L31M & RS & L31M & $\mathrm{R}$ & L31M & $\mathrm{R}$ & L31M & SB & / \\
\hline 685 & $1 a$ & Q30H e Y93H & $\mathrm{R}$ & Q30H e Y93H & $\mathrm{R}$ & Q30H e Y93H & $\mathrm{R}$ & Q30H e Y93H & $\mathrm{R}$ & Q30H \\
\hline 762 & $1 \mathrm{a}$ & Q30R & $\mathrm{R}$ & Q30R & $\mathrm{R}$ & Q30R & $\mathrm{R}$ & Q30R & $\mathrm{R}$ & Q30H \\
\hline 783 & $1 b$ & L31M & RS & L31M & $\mathrm{R}$ & L31M & $\mathrm{R}$ & L31M & SB & I \\
\hline 787 & $1 b$ & Y93H & $\mathrm{R}$ & Y93H & $\mathrm{R}$ & Y93H & $\mathrm{R}$ & Y93H & $\mathrm{R}$ & / \\
\hline 821 & $1 b$ & Y93H & $\mathrm{R}$ & Y93H & $\mathrm{R}$ & P58R e Y93H & $\mathrm{R}$ & P58R e Y93H & $\mathrm{R}$ & / \\
\hline 830 & $1 b$ & L31F & SB & L31F & $\mathrm{R}$ & L31F & SB & L31F & RS & / \\
\hline 861 & $1 \mathrm{a}$ & H58P, Y93S & SB & H58P, Y93S & SB & H58P, Y93S & SB & H58P, Y93S & $\mathrm{R}$ & H58P, \\
\hline 866 & $1 b$ & Y93H & $\mathrm{R}$ & Y93H & $\mathrm{R}$ & Y93H & $\mathrm{R}$ & Y93H & $\mathrm{R}$ & / \\
\hline 940 & $1 b$ & Y93H & $\mathrm{R}$ & Y93H & $\mathrm{R}$ & Y93H & $\mathrm{R}$ & Y93H & $\mathrm{R}$ & / \\
\hline 960 & $1 b$ & Y93H & $\mathrm{R}$ & Y93H & $\mathrm{R}$ & Y93H & $\mathrm{R}$ & Y93H & $\mathrm{R}$ & I \\
\hline 964 & $1 \mathrm{a}$ & L31M & $\mathrm{R}$ & L31M & $\mathrm{R}$ & L31M & $\mathrm{R}$ & L31M & SB & L31M \\
\hline 967 & $1 b$ & Y93N & $\mathrm{R}$ & Y93N & $\mathrm{R}$ & Y93N & $\mathrm{R}$ & Y93N & $\mathrm{R}$ & I \\
\hline 972 & $1 \mathrm{a}$ & L31M & $\mathrm{R}$ & L31M & $\mathrm{R}$ & L31M & $\mathrm{R}$ & L31M & SB & L31M \\
\hline 977 & $1 \mathrm{a}$ & H58P & SB & H58P & SB & P32L,H58P & $\mathrm{R}$ & P32L,H58P & SB & P32L \\
\hline 987 & $1 b$ & L31M & RS & L31M & $\mathrm{R}$ & L31M & $\mathrm{R}$ & L31M & SB & / \\
\hline
\end{tabular}




\begin{tabular}{|llllllllll}
1040 & $1 \mathrm{~b}$ & $\mathrm{Y93N}$ & $\mathrm{R}$ & $\mathrm{Y93N}$ & $\mathrm{R}$ & $\mathrm{Y93N}$ & $\mathrm{R}$ & $\mathrm{Y93N}$ & $\mathrm{R}$ \\
\hline 1065 & $1 \mathrm{a}$ & $\mathrm{Q} 30 \mathrm{R}$ & $\mathrm{R}$ & $\mathrm{Q} 30 \mathrm{R}$ & $\mathrm{R}$ & $\mathrm{Q} 30 \mathrm{R}$ & $\mathrm{R}$ & $\mathrm{Q}$ & $\mathrm{R}$ \\
\hline 1073 & $1 \mathrm{~b}$ & $\mathrm{Y93H}$ & $\mathrm{R}$ & $\mathrm{Y93H}$ & $\mathrm{R}$ & $\mathrm{Y93H}$ & $\mathrm{R}$ & $\mathrm{Y93H}$ & $\mathrm{R}$ \\
\hline
\end{tabular}

R: Resistance; RS: Reduced Susceptibility; S: Susceptible; SB: Substitution

Table 4: Frequency and percentage of RAS in the NS5A region, according to each drug. Adapted from Santos et al. [13].

\begin{tabular}{|lllllll|}
\hline & Drugs & & & & & \\
\hline RAS* & Daclatasvir & Elbasvir & Ledipasvir & Velpatasvir & Pibrentasvir & Ombitasvir \\
\hline L31M & N (\%) & $\mathbf{N}(\%)$ & $\mathbf{N}(\%)$ & $\mathbf{N}(\%)$ & $\mathbf{N}(\%)$ & $\mathbf{N}(\%)$ \\
\hline Y93N & $4(12.5)$ & $12(28.6)$ & $11(26.9)$ & $0(0.0)$ & $0(0.0)$ & $0(0.0)$ \\
\hline Q30R & $2(6.2)$ & $2(4.8)$ & $2(4.9)$ & $2(8.0)$ & $2(100.0)$ & $2(6.8)$ \\
\hline Y93S & $3(9.4)$ & $3(7.1)$ & $3(7.3)$ & $0(0.0)$ & $0(0.0)$ & $3(10.0)$ \\
\hline H58P + Y93S & $0(0.0)$ & $0(0.0)$ & $0(0.0)$ & $0(0.0)$ & $0(0.0)$ & $1(3.3)$ \\
\hline Q30H + Y93H & $1(3.2)$ & $1(2.3)$ & $1(2.4)$ & $1(4.0)$ & $0(0.0)$ & $1(3.3)$ \\
\hline P32L + H58P & $0(0.0)$ & $0(0.0)$ & $1(2.4)$ & $0(0.0)$ & $0(0.0)$ & $0(0.0)$ \\
\hline A92T + Y93H & $2(6.2)$ & $0(0.0)$ & $2(4.9)$ & $0(0.0)$ & $0(0.0)$ & $0(0.0)$ \\
\hline Y93H & $20(62.5)$ & $22(52.4)$ & $18(43.9)$ & $22(88.0)$ & $0(0.0)$ & $21(70.0)$ \\
\hline L31F & $0(0.0)$ & $2(4.8)$ & $0(0.0)$ & $0(0.0)$ & $0(0.0)$ & $0(0.0)$ \\
\hline L31M + P58S & $0(0.0)$ & $0(0.0)$ & $1(2.4)$ & $0(0.0)$ & $0(0.0)$ & $0(0.0)$ \\
\hline P58R + Y93H & $0(0.0)$ & $0(0.0)$ & $2(4.9)$ & $0(0.0)$ & $0(0.0)$ & $1(3.3)$ \\
\hline
\end{tabular}

*resistance associated substitutions 